\title{
Using Automated Live Cell Imaging to Reveal Early Changes during Human Motor Neuron Degeneration
}

\author{
(1)Hye Young Shin, ${ }^{1}$ Kathleen L. Pfaff, ${ }^{1}$ 'Dance S. Davidow, ${ }^{1}{ }^{\circledR}$ Chicheng Sun, ${ }^{1}$ Takayuki Uozumi, ${ }^{2}$ \\ Fumiki Yanagawa, ${ }^{2}$ Yoichi Yamazaki, ${ }^{2}$ (D) Yasujiro Kiyota, ${ }^{2}$ and ${ }^{-}$Lee L. Rubin ${ }^{1}$
}

DOI:http://dx.doi.org/10.1523/ENEURO.0001-18.2018

${ }^{1}$ Department of Stem Cell and Regenerative Biology and Harvard Stem Cell Institute, Harvard University, Cambridge, MA, United States and ${ }^{2}$ Healthcare Business Unit, Nikon Corporation, Tokyo, Japan

\section{Visual Abstract}

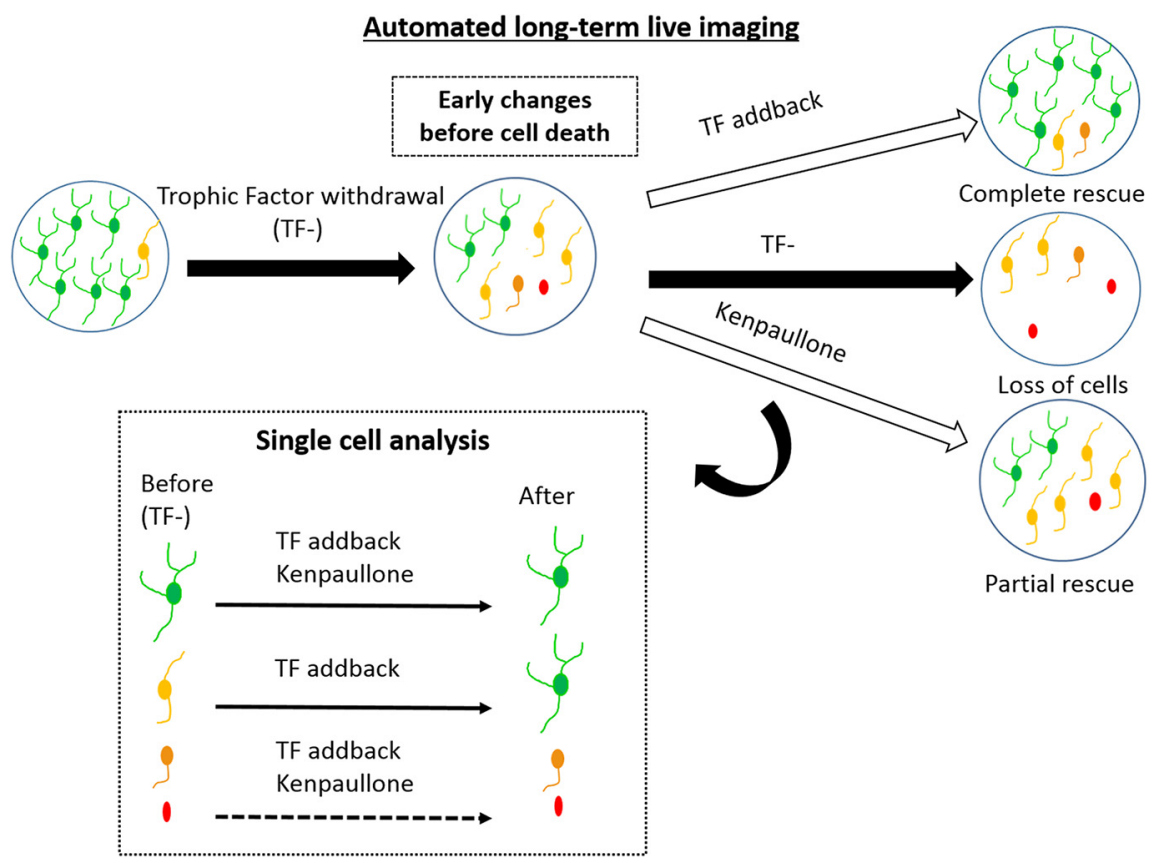

Human neurons expressing mutations associated with neurodegenerative disease are becoming more widely available. Hence, developing assays capable of accurately detecting changes that occur early in the disease

\section{Significance Statement}

This study establishes a new automated live-cell imaging method that was used to analyze human motor neurons in the process of dying after trophic factor withdrawal. Our aim was to provide an alternative to traditional endpoint survival assays by determining morphologic changes that predate death. This was accomplished by tracking large numbers of individual motor neurons over long periods of time. We identified features of motor neurons that distinguish between those neurons that can fully recover from the early stages of degeneration and those that are committed to death. Our research is of clear interest to neuroscientists interested in disease and improved methods for discovering effective therapeutics. 
process and identifying therapeutics able to slow these changes should become ever more important. Using automated live-cell imaging, we studied human motor neurons in the process of dying following neurotrophic factor withdrawal. We tracked different neuronal features, including cell body size, neurite length, and number of nodes. In particular, measuring the number of nodes in individual neurons proved to be an accurate predictor of relative health. Importantly, intermediate phenotypes were defined and could be used to distinguish between agents that could fully restore neurons and neurites and those only capable of maintaining neuronal cell bodies. Application of live-cell imaging to disease modeling has the potential to uncover new classes of therapeutic molecules that intervene early in disease progression.

Key words: Automated live time-lapse imaging instrument; heterogeneity; live cell time-lapse imaging; morphometric analysis; single cell tracking

\section{Introduction}

Technical advances in the use of human embryonic stem cell (hESC) and induced pluripotent stem cell (iPSC) technology now allow the production of human cells, especially discrete populations of neurons, for use in disease modeling, regenerative therapy, and drug screening (Grskovic et al., 2011). When compared to either standard transgenic mouse models or postmortem human brains, this provides an unprecedented opportunity to study neurodegenerative diseases such as Alzheimer's disease (AD), Parkinson's disease (PD) and amyotrophic lateral sclerosis (ALS; Brennand et al., 2015).

In some recent studies on ALS, survival assays using in vitro-derived motor neurons (MNs) from mouse ESCs, hESCs, and patient-derived iPSCs have been employed productively (Yang et al., 2013; Rodriguez-Muela et al., 2017). Nonetheless, these studies present their own sets of challenges. Cell death assays are generally simple, fixed time point counts of cell number, but current differentiation methods often result in heterogeneous mixtures of neuronal and nonneuronal cell types, each with a variable duration of cell survival. Thus, endpoint measurements, which typically measure average changes at a single time point in heterogeneous populations, do not capture the full range of complex biological responses in cells (Arrasate and Finkbeiner, 2005; Skylaki et al., 2016;

Received December 20, 2017; accepted May 12, 2018; First published June 18, 2018.

The authors declare no competing financial interests.

Author contributions: H.Y.S., K.L.P., L.S.D., C.S., and L.L.R designed experiments and evaluated results; H.Y.S. performed all experiments; T.U., F.Y., Y.Y., and Y.K. designed and applied live cell morphometric analysis; H.Y.S., K.L.P., and L.L.R. wrote manuscript.

This research was supported by a sponsored research grant from Nikon Corporation to LLR.

Acknowledgments: We thank Professor Kenneth Chien for providing the H9-Islet1::GFP cell line. We thank Kara Held and Naomi Okugawa for experimental support, Silvia Piccinotti and Tim Ahfeldt for helpful advice, and Richard Krolewski and Jane Lalonde for critical review of this manuscript. Thank you to Brandi Mattson of Life Science Editors for revisions and suggestions.

Address correspondence to Lee Rubin, PhD, Dept of Stem Cell and Regenerative Biology, Harvard University, Director of Translational Medicine, Harvard Stem Cell Institute, Sherman Fairchild G60, 7 Divinity Ave., Cambridge, MA 02138. E-mail: lee_rubin@harvard.edu.

DOl:http://dx.doi.org/10.1523/ENEURO.0001-18.2018

Copyright $\odot 2018$ Shin et al.

This is an open-access article distributed under the terms of the Creative Commons Attribution 4.0 International license, which permits unrestricted use, distribution and reproduction in any medium provided that the original work is properly attributed.
Rodriguez-Muela et al., 2017). Furthermore, while it is well known that producing neurons from human pluripotent cells can be time consuming - often a period of monthsthe amount of time required for diseased neurons to die can also be quite protracted.

Time-lapse imaging of individual live cells offers an exciting alternative to address these limitations (Skibinski and Finkbeiner, 2013; Skylaki et al., 2016). This approach also affords an examination of early disease phenotypes, when therapeutic treatments are most effective in prolonging survival and minimizing disease progression. Neuronal morphology can reflect neuronal function and health. Morphologic changes, such as neuronal shrinkage and dendrite retraction, have been reported in postmortem patient samples of AD, PD, and ALS (Davies et al., 1987; Kiernan and Hudson, 1993; Anglade et al., 1997). In particular, surviving MNs in ALS human postmortem tissues show significantly decreased size compared to MNs in healthy tissue, and cell size positively correlates with the number of surviving MNs (Kiernan and Hudson, 1993). Early changes in the properties of axons and dendrites are also a consistent feature of neurodegenerative diseases (Davies et al., 1987; Kiernan and Hudson, 1993; Anglade et al., 1997). So, we hypothesized that defining the earliest stress or disease-related morphologic changes, such as cell body size, neurite length, and number of nodes (also called roots) in cultured human neurons will guide the development of assays to identify drugs that might have the greatest therapeutic efficacy.

In this study, we defined nodes as the location on the cell body from which the neurites project. We focused on node number as a key parameter for neuronal fitness for both practical and technical reasons. The practical reason was that, as mentioned above, neurodegenerative diseases have varying forms of dendritic pathology including loss of neurites, which would be reflected by decreased number of nodes. Counting and tracking nodes is therefore a surrogate measurement for phenotypes found in pathologic specimens from neurodegenerative disease patients. The technical reason centered on reducing the complexity of analyses that depend on the identification and tracking of individual neurites.

Here, we perform a computational, single-cell analysis of in vitro-derived human MNs that were individually observed in large numbers for more than 2 weeks to identify key morphometric changes that occur during the onset and reversal of neuronal degeneration leading to cell death. We employed trophic factor (TF) withdrawal as a 
well-known stressor to study the early processes that underlie MN death. We also measured to ability of TF addback to rescue neurons at various stages of degeneration. Finally, the effects of kenpaullone, previously identified in a MN survival screen (Yang et al., 2013), were compared with effects of TF addback. We used the BioStation CT, a self-contained unit that includes an incubator, a robotic arm, and a microscope, to study the early processes that underlie MN death. Our work was enabled by the development of imaging software that identifies individual MNs and tracks them over time, even when they shift position.

\section{Materials and Methods}

\section{Motor neuron production}

All experiments with hESCs were reviewed and approved by Harvard University Embryonic Stem Cell Oversight Committee. The H9-Islet1::GFP cell line (female human hESCs) was confirmed to be mycoplasma negative by PCR and ELISA. MNs were differentiated from hESCs and grown on Matrigel (Corning) with mTeSR (Stem Cell Technologies), for 21 days. A two-step "hybrid method" was devised in which cells were first maintained on dishes in 2D and later switched to 3D embryoid bodies (EBs). Multipotent neural stem cells (NSCs) were derived from hESCs in 2D. For 2D culture, Accutase-dissociated single hESCs were seeded at a density of $4 \times 10^{6}$ cells with $\mathrm{mTeSR}$ media onto coated $10-\mathrm{cm}$ plates (Corning). Vitronectin (Stem Cell Technologies, $10 \mu \mathrm{g} / \mathrm{ml}$ ), laminin (Life Technologies, $10 \mu \mathrm{g} / \mathrm{ml}$ ), and fibronectin (BD Biosciences, $10 \mu \mathrm{g} / \mathrm{ml}$ ) were used for coating. Neural differentiation was induced with basic fibroblast growth factor (bFGF) and dual SMAD inhibition for 7 days (NIM: DMEM/ F12 with N2 (Life Technologies), B27 (Life Technologies), Dorsomorphin (Stemgent, $1 \mu \mathrm{M}$ )/SB431542 (Stemgent, 1 $\mu \mathrm{M}) / \mathrm{bFGF}$ (Life Technologies, $10 \mathrm{ng} / \mathrm{ml})$. Three days after plating, retinoic acid (RA; Sigma Aldrich, $3 \mu \mathrm{M}$ ) and brainderived neurotrophic factor (BDNF; R\&D Systems, $30 \mathrm{ng} /$ $\mathrm{ml}$ ) were added. Smoothened agonist (SAG 1.3; DNSK International, $1 \mu \mathrm{M}$ ) was added at day 5. For 3D cultures, day 8 NSCs were dissociated with Accutase and cultured at a density of $400,000 / \mathrm{ml}$ on low-adherence flasks in suspension medium containing ROCK inhibitor $(10 \mu \mathrm{M})$ and bFGF $(10 \mathrm{ng} / \mathrm{ml})$ to generate EBs. Spherical aggregates of NSCs were maintained up to day 21 with RA (1 $\mu \mathrm{M})$, SAG $1.3(1 \mu \mathrm{M})$, BDNF (10 $\mathrm{ng} / \mathrm{ml})$, glial cell-derived neurotrophic factor (GDNF; R\&D Systems, $10 \mathrm{ng} / \mathrm{ml}$ ), insulin-like growth factor (IGF; R\&D Systems, $10 \mathrm{ng} / \mathrm{ml}$ ), cAMP (Santa Cruz Biotechnology, $0.1 \mu \mathrm{M}$ ), and dATP (Santa Cruz Biotechnology, $1 \mu \mathrm{M}$ ). AraC (Sigma Aldrich, 2 $\mu \mathrm{M})$ was added from day 17 to 21 to eliminate proliferating cells.

In this study, assay development was necessary to determine the optimal cell density to achieve uniform distribution of MNs. We tested the robustness of the experimental system across multiple biological replicates using independent batches of MNs. There is some interexperimental variability, which is a combined consequence of variability in the differentiation efficiency, differential cell survival during dissociation, and deviations in the plating cell densities. Despite this, four (Fig. 1) and five (Figs. 2-6) independent batches of MN differentiation exhibited remarkably consistent trends in analyzed results, indicating that this model is reliable and reproducible.

\section{Treatment of cells}

Withdrawal of trophic factors [TFs; BDNF, GDNF, and ciliary neurotrophic factor (CNTF)] is a well-established method to activate neuronal apoptosis (Yang et al., 2013). To initiate cell death in our cultures, we withdrew TF support along with B27 and N2 supplements from MNs $\left(\mathrm{TF}^{-}\right)$at day 1 (1 day after live imaging initiation). To study the early processes that underlie MN death by TF withdrawal, as well as to distinguish different actions of kenpaullone and TF addback treatment on the MNs deprived of TF at day 1, TFs (BDNF, GDNF, CNTF, B27, and N2) were reintroduced to the cultures (defined as "TF addback") at varying lengths of time (6, 7, or 8 days) after their withdrawal. For kenpaullone treatment, two different concentrations (2.5 and $5 \mu \mathrm{M})$ were supplied to MNs during the entire period in which they were maintained in the absence of TF.

\section{Assay development for automated live time-lapse imaging}

To prepare MNs for live imaging, day 21 EBs were dissociated with Accutase, triturated until no clumps were visible, and seeded into 96-well $\mu$ Clear black-walled plates (Greiner Bio-One; Cat \# 655090) with primary mouse glia as feeder cells, and maintained with BDNF (10 $\mathrm{ng} / \mathrm{ml})$, GDNF (10 ng/ml), and CNTF (10 ng/ml). FluoroBrite DMEM (Thermo Fisher Scientific) medium with N2 and B27 was used to minimize background fluorescence.

Cell density is a critical variable in neuronal cultures and for in vitro live-cell imaging. We first evaluated how MNs plated at different initial densities performed in our survival assay. We compared cells plated at $3.5 \times 10^{4}(35 \mathrm{~K})$ cells and at $6.0 \times 10^{4}(60 \mathrm{~K})$ cells per well. We tracked an average of 110 individual GFP-positive MNs in the $35 \mathrm{~K} /$ well plating density and $140 \mathrm{MNs}$ per well in the $60 \mathrm{~K} /$ well plating density (data not shown). We noted that the number of MNs that were able to be tracked did not increase linearly with the number of cells plated. This reflects an inherent challenge with automated live cell tracking, which is that cells are more difficult to individually distinguish and track as they become more crowded. When plating at a lower cell density onto a glial support layer, we observed that MNs exhibit little clumping, which yields a single neuron distribution for more accurate tracking and measurements. Therefore, a $35 \mathrm{~K} /$ well plating density was employed for all subsequent experiments.

\section{Automated time-lapse live imaging}

Three technical replicate wells per condition per experiment (four biological replicates for Fig. 1; five biological replicates for Figs. 2-6) were imaged. A 10× objective was used to acquire phase and fluorescent images at each time point. For each well, a $4 \times 4$ stitched tiling captured an area of $3.08 \times 3.08 \mathrm{~mm}$, which covers $\sim 30 \%$ 
A

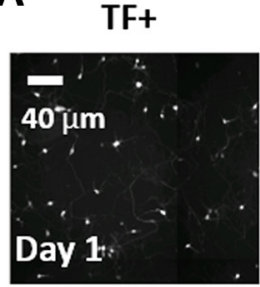

TF-

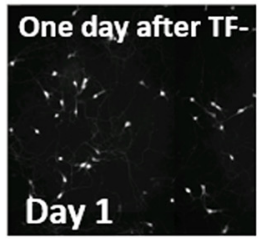

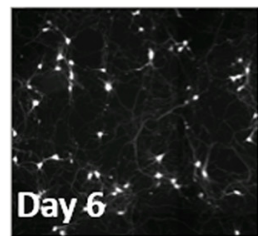

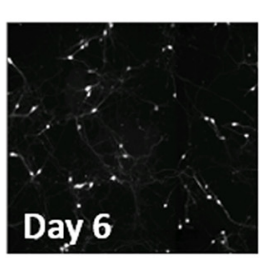

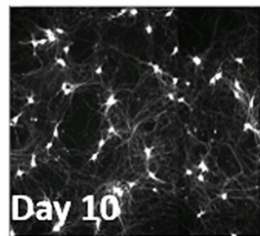

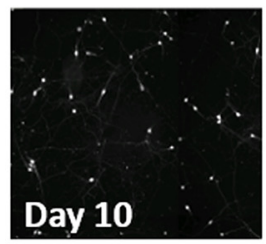

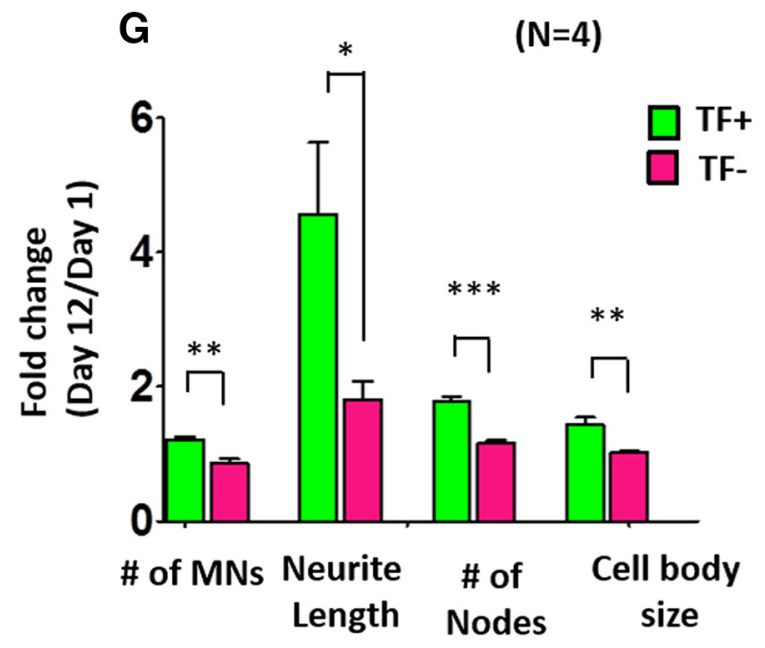

E
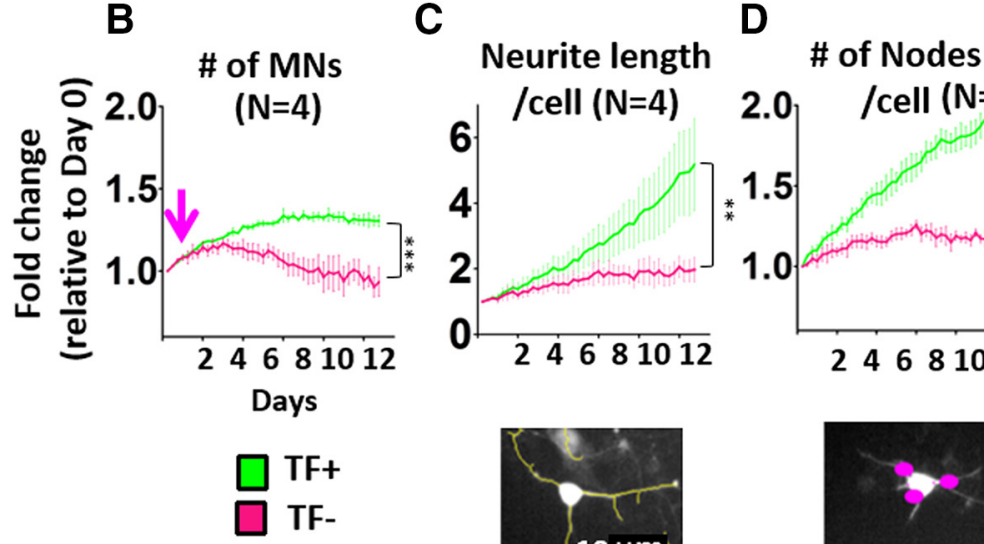

C
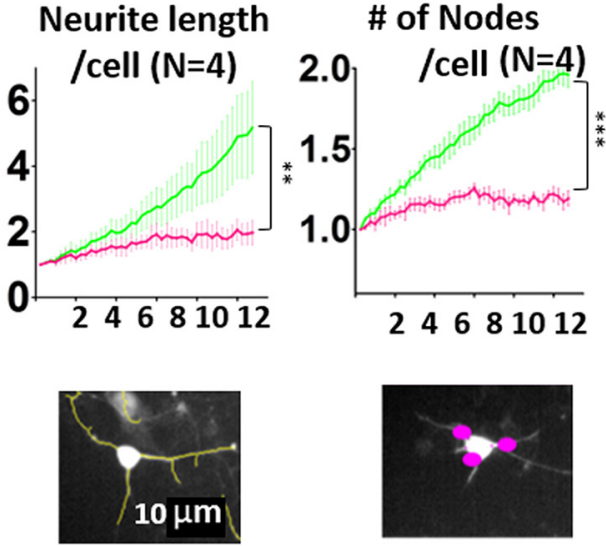
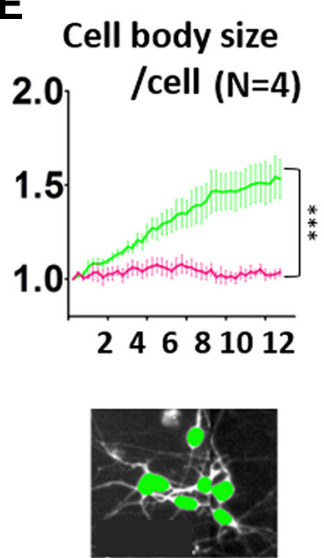

$\mathbf{F}$

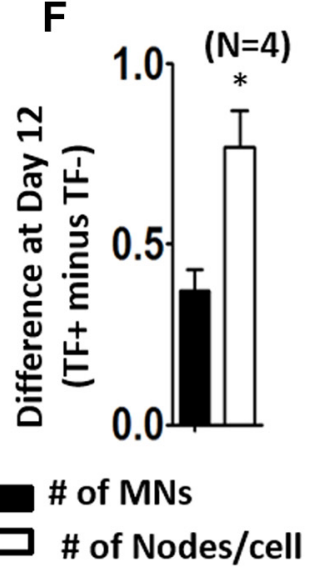

Figure 1. Identifying morphologic changes that precede neuronal death using computational quantitation. $\boldsymbol{A}$, Representative BioStation CT images of human Islet1::GFP-positive MNs in basal $\left(\mathrm{TF}^{+}\right)$and withdrawal $\left(\mathrm{TF}^{-}\right)$conditions at key experimental time points: day 1 (1 day after TF withdrawal), day 6 , and day 10 of imaging (scale bar $=40 \mu \mathrm{m}$ ). $\boldsymbol{B}-\boldsymbol{E}$, Time series plots comparing the populations of Islet1::GFP MNs in $\mathrm{TF}^{+}$(green) and $\mathrm{TF}^{-}$(magenta) conditions. Quantitation was done at each time point for the number $(\#)$ of MNs $(\boldsymbol{B})$, neurite length per cell $(\boldsymbol{C})$, number of nodes per cell $(\boldsymbol{D})$, and cell body size per cell $(\boldsymbol{E})$. The arrow indicates time of TF withdrawal (day 1). The average fold change relative to day 0 for all of the measured parameters is shown. A significant different between $\mathrm{TF}^{+}$and $\mathrm{TF}^{-}$over time was found by two-way repeated-measures ANOVA after Bonferroni correction in the number of MNs $(p<0.001, F=13.63$, DFn $=1 ; \boldsymbol{B})$, neurite length per cell $(p<0.01, F=5.27$, DFn $=1 ; \boldsymbol{C})$, number of nodes per cell $(p<0.001$, $F=30.57, \mathrm{DFn}=1 ; \boldsymbol{D})$ and cell body size per cell $(p<0.001, F=11.54$, DFn $=1 ; \boldsymbol{E})$, and significant differences between $\mathrm{TF}^{+}$and $\mathrm{TF}^{-}$were observed at the end point by Bonferroni post-tests: $* *, p<0.01 ; * * *, p<0.001$. Data presented as mean + SEM. $(n=4$ biological replicate experiments, each with three technical replicates.) $\boldsymbol{F}$, Comparison of the effect magnitudes between $\mathrm{TF}^{+}$and $\mathrm{TF}^{-}$ for number of MNs $(\boldsymbol{B})$ and number of nodes per cell $(\boldsymbol{D})$ at day 12 . Data presented as mean $+\mathrm{SEM}$. $*, p<0.05$ by $t$ test $(n=4$ biological replicate experiments, each with three technical replicates). G, Extracted time series data showing fold change measurements in features between day 1 and day 12. Data presented as mean + SEM. $*, p<0.05 ; * *, p<0.01 ; * * *, p<0.001$ by $t$ test ( $n=4$ biological replicate experiments, each with three technical replicates).

of the total growth area of the well. Images were automatically captured every 6 hours for $14-17$ days.

\section{Cell tracking and image analysis}

All image analysis was performed using CL-Quant software (Nikon Corporation), and Nikon designed specialized viewer software to easily review the analysis results from these data sets. To detect the morphologic properties of the cells in the culture, morphologic filters (or masks) were designed to identify and quantify the different features, including cell body number, neurite length, cell body size, compactness, and intensity, in the raw image. To detect nodes, a combination of the cell body filter and neurite filter was used to detect the overlapping regions between neurite and cell body. This filter combination was then used to identify nodes in the raw images. After the filters for detection of each cell were optimized, the tracking was performed using the filter for cell body. The setting parameters used in CL-Quant for this tracking are as follows: minimum object size: 50, maximum object size: 999999, maximum search range: 200 , split threshold: 0.90 , merge threshold: 0.70 , minimum trajectory length: 5 , object split: ignore split, object merge: merge with partition, enable lineage: off, enable robust measure- 
Table 1. Numbers of tracked MNs to evaluate cell class transitions following TF addback (day 6-14)

\begin{tabular}{|c|c|c|c|c|c|c|c|c|c|}
\hline & \multirow[b]{2}{*}{$\begin{array}{l}\text { Total tracked } \\
\text { \# of MNs }\end{array}$} & \multicolumn{4}{|c|}{ Outcome of fate transition } & \multirow[b]{2}{*}{$\begin{array}{l}\text { Tracked \# of } \\
\text { Class B MNs }\end{array}$} & \multicolumn{3}{|c|}{ Outcome of fate transition } \\
\hline & & $\begin{array}{l}\text { Tracked \# of } \\
\text { Class A MNs }\end{array}$ & Class $A$ to $A$ & Class $A$ to $B$ & Class $A$ to $C$ & & Class $B$ to $A$ & Class $B$ to $B$ & Class $\mathrm{B}$ to $\mathrm{C}$ \\
\hline $\mathrm{TF}^{+}$ & $110.9 \pm 32.05$ & $83.33 \pm 30.16$ & $62.27 \pm 22.07$ & $2.267 \pm 1.024$ & $18.80 \pm 7.240$ & $27.57 \pm 4.540$ & $9.533 \pm 2.421$ & $3.300 \pm 0.9638$ & $14.73 \pm 2.379$ \\
\hline TF addback at day 6 & $93.60 \pm 29.36$ & $58.33 \pm 24.68$ & $40.17 \pm 16.36$ & $2.033 \pm 1.311$ & $16.13 \pm 7.285$ & $35.27 \pm 6.954$ & $10.43 \pm 2.834$ & $3.167 \pm 0.7853$ & $21.67 \pm 4.001$ \\
\hline TF addback at day 7 & $97.97 \pm 28.09$ & $46.92 \pm 16.25$ & $26.56 \pm 11.81$ & $1.333 \pm 0.5164$ & $19.03 \pm 4.254$ & $44.67 \pm 11.90$ & $33.90 \pm 17.07$ & $2.100 \pm 0.9422$ & $21.27 \pm 6.517$ \\
\hline TF addback at day 8 & $110.3 \pm 31.51$ & $57.27 \pm 24.46$ & $33.90 \pm 17.07$ & $2.100 \pm 0.9422$ & $21.27 \pm 6.517$ & $55.54 \pm 11.60$ & $25.83 \pm 13.03$ & $5.600 \pm 1.431$ & $24.43 \pm 6.128$ \\
\hline $\mathrm{TF}^{-}$ & $101.6 \pm 30.11$ & $55.87 \pm 19.52$ & $25.83 \pm 13.03$ & $5.600 \pm 1.431$ & $24.43 \pm 6.128$ & $45.73 \pm 12.47$ & $5.267 \pm 2.125$ & $10.70 \pm 4.803$ & $29.77 \pm 6.136$ \\
\hline
\end{tabular}

Data are presented as mean $\pm \operatorname{SEM}(n=5)$.

Table 2. Numbers of tracked MNs to evaluate cell class transitions during kenpaullone treatment (day 0-14)

\begin{tabular}{|c|c|c|c|c|c|c|c|c|c|}
\hline \multirow{3}{*}{$\begin{array}{l}\text { Conditions } \\
\mathrm{TF}^{+}\end{array}$} & \multirow[b]{2}{*}{$\begin{array}{l}\text { Total tracked } \\
\# \text { of MNs }\end{array}$} & \multicolumn{5}{|c|}{ Outcome of fate transition } & \multicolumn{3}{|c|}{ Outcome of fate transition } \\
\hline & & $\begin{array}{l}\text { Tracked \# of } \\
\text { Class A MNs }\end{array}$ & Class $A$ to $A$ & Class $A$ to $B$ & Class $\mathrm{A}$ to $\mathrm{C}$ & $\begin{array}{l}\text { Tracked \# of } \\
\text { Class B MNs }\end{array}$ & Class $B$ to $A$ & Class $\mathrm{B}$ to $\mathrm{B}$ & Class $\mathrm{B}$ to $\mathrm{C}$ \\
\hline & $86.20 \pm 17.08$ & $42.47 \pm 13.86$ & $27.47 \pm 10.07$ & $0.6000 \pm 0.2449$ & $14.40 \pm 4.128$ & $43.73 \pm 6.322$ & $14.87 \pm 4.121$ & $1.733 \pm 0.5907$ & $27.13 \pm 3.023$ \\
\hline $\mathrm{TF}^{+} /$Ken $5 \mathrm{uM}$ & $80.27 \pm 19.01$ & $41.07 \pm 15.52$ & $27.60 \pm 11.26$ & $0.7333 \pm 0.3399$ & $12.73 \pm 4.153$ & $39.20 \pm 6.538$ & $17.00 \pm 4.791$ & $2.333 \pm 0.5055$ & $19.87 \pm 2.277$ \\
\hline $\mathrm{TF} /$ Ken $2.5 \mathrm{uM}$ & $98.27 \pm 21.23$ & $42.80 \pm 16.05$ & $21.40 \pm 8.362$ & $2.400 \pm 0.8781$ & $19.00 \pm 7.551$ & $55.47 \pm 10.46$ & $15.07 \pm 4.993$ & $7.000 \pm 1.623$ & $33.40 \pm 5.499$ \\
\hline TF-/Ken 5uM & $96.87 \pm 16.16$ & $38.27 \pm 11.93$ & $18.67 \pm 5.544$ & $1.867 \pm 0.7196$ & $17.73 \pm 5.743$ & $58.60 \pm 12.21$ & $11.80 \pm 4.196$ & $7.200 \pm 2.560$ & $39.60 \pm 7.318$ \\
\hline $\mathrm{TF}^{-}$ & $92.53 \pm 19.25$ & $42.47 \pm 14.90$ & $11.13 \pm 3.868$ & $4.467 \pm 1.698$ & $26.87 \pm 9.443$ & $50.07 \pm 6.998$ & $6.867 \pm 1.695$ & $4.333 \pm 1.038$ & $38.87 \pm 5.904$ \\
\hline
\end{tabular}

Data are presented as mean $\pm \operatorname{SEM}(n=5)$.

Table 3. Summary of statistical analyses

\begin{tabular}{|c|c|c|c|c|}
\hline Location & Description & Data structure & Type of test & Statistical value \\
\hline \multirow[t]{2}{*}{ a } & Fig. $1 B-E$ & $\begin{array}{l}\text { Normal (Gaussian distribution) } \\
\text { at the end point; number } \\
\text { (n) of biological } \\
\text { experiments }=4 \text { with three } \\
\text { technical replicates }\end{array}$ & $\begin{array}{l}\text { Two-way repeated-measures } \\
\text { ANOVA after Bonferroni } \\
\text { correction }\end{array}$ & $\begin{array}{l}\text { Number (\#) of MNs : } p<0.001, F=13.63, \mathrm{DFn}=1 \text {; Neurite length per cell: } p<0.01 \\
F=5.27, \mathrm{DFn}=1 ; \# \text { of Nodes per cell }: p<0.001, F=30.57, \mathrm{DFn}=1 \text {; Cell body } \\
\text { size per cell }: p<0.001, F=11.54, \mathrm{DFn}=1\end{array}$ \\
\hline & & & $\begin{array}{l}\text { Unpaired two-tailed } t \text {-test at } \\
\text { the end point }\end{array}$ & $\begin{array}{l}\text { \# of MNs : } p<0.001 \text {; Neurite length per cell: } p<0.01 \text {; \# of Nodes per cell : } p< \\
0.001 \text {; Cell body size per cell }: p<0.001\end{array}$ \\
\hline b & Fig. $1 F$ & Normal, $n=4$ & Unpaired two-tailed $t$-test & $p<0.05$ \\
\hline c & Fig. $1 G$ & Normal, $n=4$ & Unpaired two-tailed $t$-test & $\begin{array}{l}\# \text { of MNs: } p<0.01 \text {, Neurite length per cell: } p<0.05 \text {; \# of Nodes per cell: } p<0.0001 \text {, } \\
\text { Cell body size: } p<0.01\end{array}$ \\
\hline d & Fig. 2C & Normal, $n=5$ & Unpaired two-tailed $t$-test & $\mathrm{TF}^{+}: p<0.001, \mathrm{TF}$ addback at day $6: p<0.05$ \\
\hline \multirow[t]{2}{*}{ e } & Fig. 2D, $E$ & $\begin{array}{l}\text { Normal at the end point; } \\
\quad n=5\end{array}$ & $\begin{array}{l}\text { Two-way repeated-measures } \\
\text { ANOVA after Bonferroni } \\
\text { correction }\end{array}$ & $\begin{array}{l}\text { Neurite length per cell: } p<0.01, F=6.555, \mathrm{DFn}=4 ; \# \text { of Nodes per cell: } p<0.05, F \\
\quad=3.356, \mathrm{DFn}=4\end{array}$ \\
\hline & & & $\begin{array}{l}\text { Unpaired two-tailed } t \text {-test at } \\
\text { the end point }\end{array}$ & $\begin{array}{l}\text { Neurite length per cell: TF addback at day } 6 \text {, day } 7 \text {, and day } 8: p<0.001 \text {, } \\
\text { respectively; \# of Nodes per cell: TF addback at day } 6 \text {, day } 7: p<0.001 \text {; TF } \\
\text { addback at day } 8: p<0.01\end{array}$ \\
\hline$f$ & Fig. 2G & Normal, $n=5$ & Unpaired two-tailed $t$-test & $\mathrm{TF}^{+}$and $\mathrm{TF}^{+} / \mathrm{Ken} 5 \mu \mathrm{M}: p<0.001 ; \mathrm{TF}^{-} / 2.5 \mu \mathrm{M}$ and $\mathrm{TF}^{-} / 5 \mu \mathrm{M}: \mathrm{p}<0.05$ \\
\hline \multirow[t]{2}{*}{ g } & Fig. $2 \mathrm{H}, \mathrm{I}$ & $\begin{array}{l}\text { Normal at the end point; } n= \\
5\end{array}$ & $\begin{array}{l}\text { Two-way repeated-measures } \\
\text { ANOVA after Bonferroni } \\
\text { correction }\end{array}$ & $\begin{array}{l}\text { Neurite length per cell: } p<0.05, F=4.01 \text {, DFn }=4 \text {; \# of Nodes per cell: not } \\
\text { significant }\end{array}$ \\
\hline & & & $\begin{array}{l}\text { Unpaired two-tailed } t \text {-test at } \\
\text { the end point }\end{array}$ & $\begin{array}{l}\text { Neurite length per cell: } \mathrm{TF}^{+} \text {and } \mathrm{TF}^{+} / \text {Ken } 5 \mu \mathrm{M}: p<0.001 ; \# \text { of Nodes per cell: } \mathrm{TF}^{+}: p \\
\quad<0.001, ; \mathrm{TF}^{+} / \text {Ken5 } \mu \mathrm{M}: p<0.01\end{array}$ \\
\hline \multirow[t]{2}{*}{ h } & Fig. 3D & $\begin{array}{l}\text { Normal at the end point; } n= \\
\quad 5\end{array}$ & $\begin{array}{l}\text { Two-way repeated-measures } \\
\text { ANOVA correction after } \\
\text { Bonferroni correction }\end{array}$ & Fold change of MNs with 4 or more nodes : $p<0.05, F=3.949, \mathrm{DFn}=2$ \\
\hline & & & $\begin{array}{l}\text { Unpaired two-tailed } t \text {-test at } \\
\text { the end point }\end{array}$ & $p<0.05$ \\
\hline i & Fig. 4C & Normal, $n=5$ & Unpaired two-tailed $t$-test & $\mathrm{TF}^{+}: p<0.001, \mathrm{TF}$ addback at day $6: p<0.001$ \\
\hline j & Fig. 4D & Normal, $n=5$ & Unpaired two-tailed $t$-test & $\mathrm{TF}^{+}: p<0.01, \mathrm{TF}^{+} /$Ken $5 \mu \mathrm{M}: p<0.01$ \\
\hline $\mathrm{k}$ & Fig. $5 \mathrm{C}$ & Normal, $n=5$ & Unpaired two-tailed $t$-test & $\begin{array}{l}\mathrm{TF}^{+}: p<0.001, \mathrm{TF} \text { addback at day } 6: p<0.001, \mathrm{TF} \text { addback at day } 7: p<0.05 \text {, } \\
\text { addback at day } 8: p<0.05\end{array}$ \\
\hline I & Fig. 5D & Normal, $n=5$ & Unpaired two-tailed $t$-test & $\begin{array}{l}\mathrm{TF}^{+}: p<0.001, \text { TF addback at day6: } p<0.001, ; \text { TF addback at day7: } p<0.05 \\
\text { addback at day8: } p<0.05\end{array}$ \\
\hline $\mathrm{m}$ & Fig. 5E & Normal, $n=5$ & Unpaired two-tailed $t$-test & $\begin{aligned} \mathrm{TF}^{+}: p<0.001, \mathrm{TF}^{+} / \text {Ken } 5 \mu \mathrm{M}: p<0.001, \mathrm{TF}^{-} / \text {Ken } 2.5 \mu \mathrm{M}: p<0.01, \mathrm{TF}^{-} / \text {Ken } 5 \mu \mathrm{M}: \\
\quad p<0.01\end{aligned}$ \\
\hline$n$ & Fig. $5 \mathrm{~F}$ & Normal, $n=5$ & Unpaired two-tailed $t$-test & $\mathrm{TF}^{+}: p<0.01, \mathrm{TF}^{+} /$Ken $5 \mu \mathrm{M}: p<0.01$ \\
\hline o & Fig. $6 \mathrm{C}$ & Normal, $n=5$ & Unpaired two-tailed $t$-test & TF addback at day 6 , day 7 , and day $8: p<0.001$, respectively \\
\hline $\mathrm{p}$ & Fig. 6D & Normal, $n=5$ & Unpaired two-tailed $t$-test & TF addback at day 6 , day 7 , and day $8: p<0.001$, respectively \\
\hline
\end{tabular}

ment: off, object-to-object overlap: off, remove short track when merging: off.

To track individual cells, a cell ID number was assigned to each identified cell body at the beginning of the imag- ing period. Comparing the cell body filters between two subsequent time frames for morphology, position, and overlap, scores were given to allow the best assignment of cell IDs (see example images in Fig. $4 A$ ). This compu- 


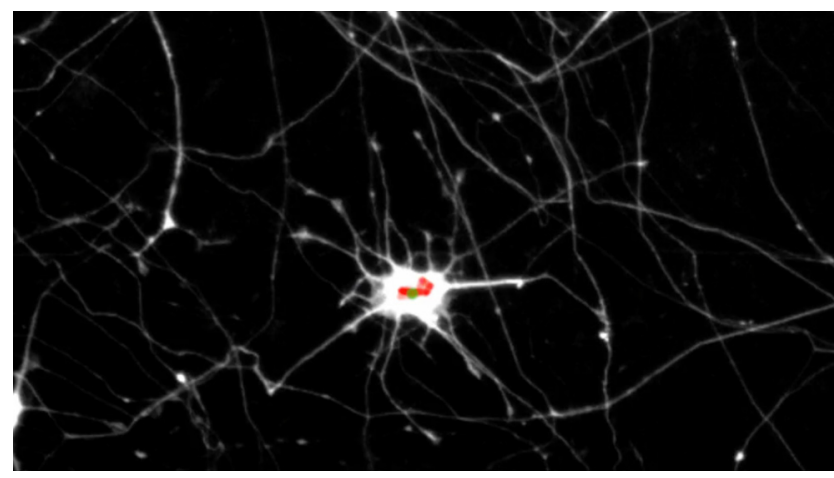

Video 1. Representative video of H9-Islet1::GFP MN, captured every 6 hours for 14 days. Single-cell tracking software successfully identifies and tracks individual MNs. Green dot indicates center of the cell body. The red line maps the MN's trajectory over time. [View online]

tational matching was performed iteratively throughout the entire time-lapse data set. By joining and tracking the cell IDs with the highest score, cell tracking was completed for all objects identified by the cell body filter.

The tracking data set for the cell body filter was then used as the basis to track the number of nodes over time. A moving average (MA) was employed for smoothing the data set before plotting. The node detection algorithm was further used to define class A MNs (with three or more nodes) and class B MNs (with one or two nodes) and to individually track MNs to determine survival outcomes. The number of MNs tracked for all TF addback and kenpaullone experiments at the $35 \mathrm{~K}$ seeding density can be found in Tables 1 and 2 .

When it was observed that TF addback rescued some class B MNs to class A MNs, we devised a reverse tracking approach to distinguish rescuable class $B$ MNs from unrescuable class $B$ MNs. Rescued and unrescued class B MNs were identified and individually tracked backward to the time point of addback (day 6,7 , or 8 ). Then, morphologic parameters such as node number, cell body size, compactness, and velocity at days 6,7 , and 8 were tallied and averaged.

\section{Statistical analysis}

Statistical analysis was performed using GraphPad Prism 5. All data (four biological replicates: Fig. 1; five biological replicates: Figs. 2-6) are presented as the mean + SEM. The experiments were not randomized. The scientists were not blinded during experiments; however, the imaging analysis was conducted in an unbiased way across all samples and was based on automated image analyses. Repeated measure-based parameters (such as neurite length per cell, number of nodes, and cell body size per cell over time) were analyzed using two-way repeated-measures ANOVA followed by Bonferroni correction, followed by $t$ test. Statistical details ( $p$ value, $F$, and degree of freedom (Dfn) are provided in Table 3 along with the results of the two-way ANOVA testing. When only two groups were analyzed, statistical significance was determined using unpaired two-tailed $t$ test. Normal (Gaussian) distribution was determined by column test. A

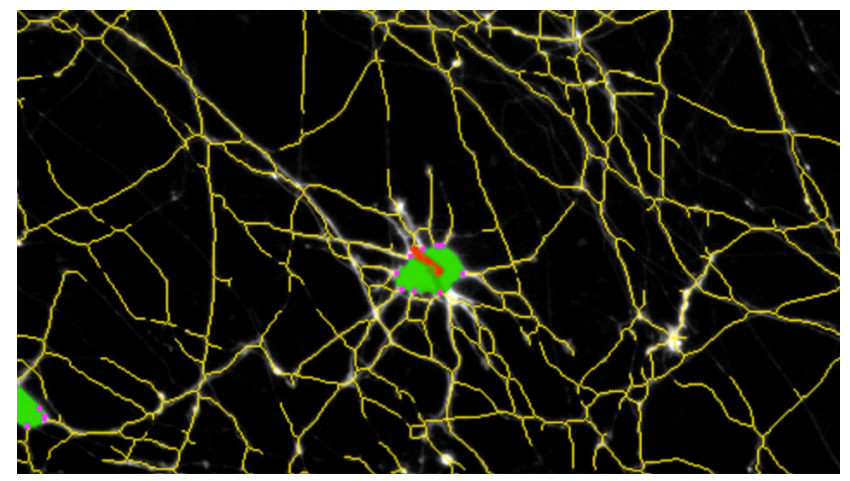

Video 2. Morphometric single-cell tracking analysis tracks neurite length (yellow), number of nodes (magenta), and cell body size (green) of MN over time. Because of the increasing complexity of the neuritic network, the number of nodes metric is better than neurite length for tracking at the single-cell level. Images were captured every 6 hours for 14 days. [View online]

$p$ value of $<0.05(*),<0.01(* *)$, and $<0.001$ (***) was used to denote statistical significance. Superscript letters listed with figure legends and $p$-values correspond to the statistical tests shown in Table 3.

\section{Results}

\section{Assay development for automated robotic live-cell imaging of MN cultures}

For experiments described here, we took advantage of the fact that there are numerous protocols available for deriving human and mouse MNs from ESCs or iPSCs, and we sought to identify the most robust differentiation method for our live-cell imaging assay. Specifically, we used an MN reporter hESC line (H9-Islet1::GFP), in which
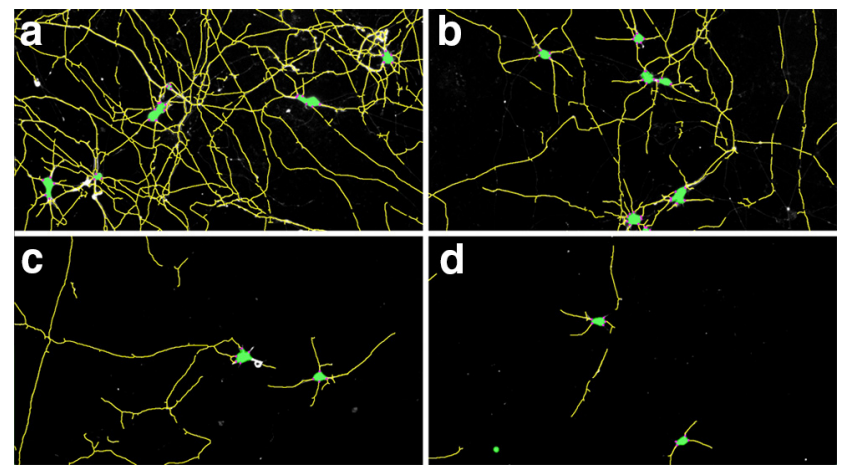

Video 3. Single-cell tracking analysis of neurite length (yellow), number of nodes (magenta), and cell body size (green) of MN in four experimental conditions: TF $(\boldsymbol{A})$, TF addback at day $6(\boldsymbol{B}), 5$ $\mu \mathrm{M}$ kenpaullone and TF withdrawal (C), and TF withdrawal (D). TF was withdrawn at day 1 (1 day after live imaging initiation), and TF was added at day 6 (C), and $5 \mu \mathrm{m}$ kenpaullone was added during the entire period in which MNs were maintained in the absence of TF. Images were captured every 6 hours for 14 days. In TF $(\boldsymbol{A})$, individual MNs increased neurite length and number of nodes, and TF addback at day $6(\boldsymbol{B})$ rescues both neurite length and number of nodes. Kenpaullone $(\boldsymbol{C})$ is less protective compared to TF addback $(\boldsymbol{B})$. In TF withdrawal $(\boldsymbol{D})$, the neurites retract and the cell body size and number of nodes decrease. [View online] 
A

\section{TF addback}

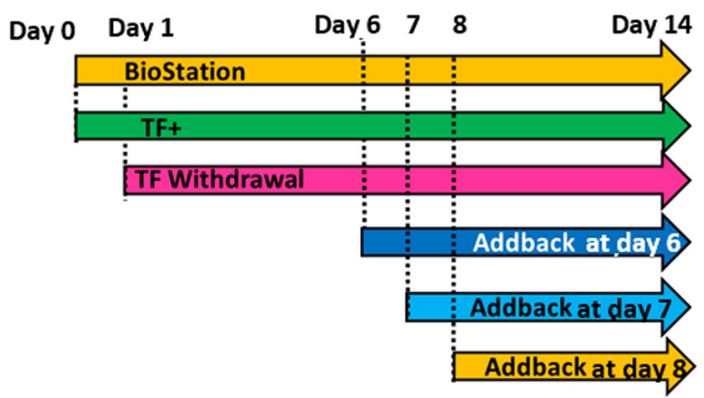

B
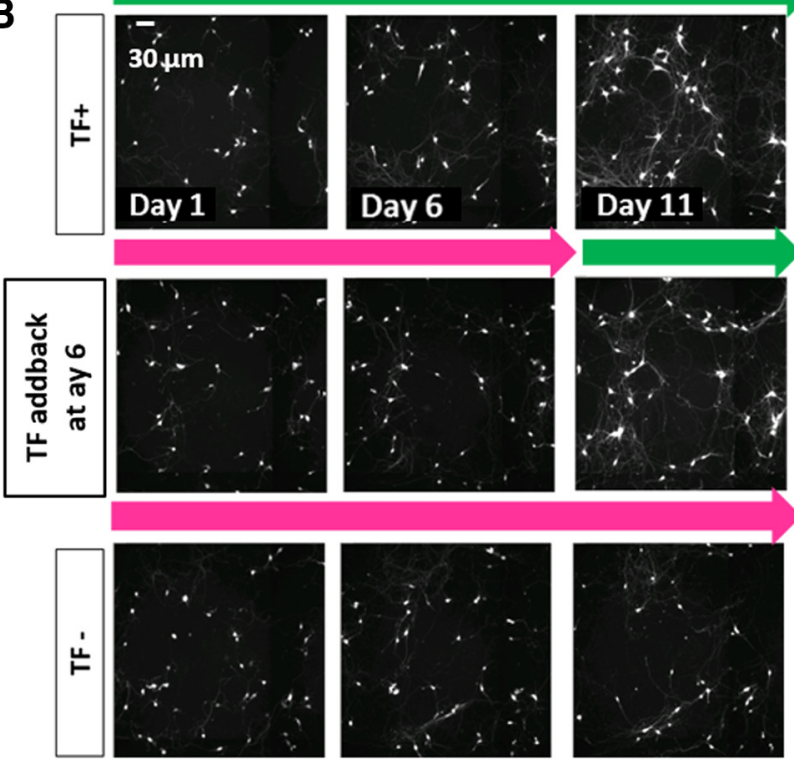

( $N=5$ )

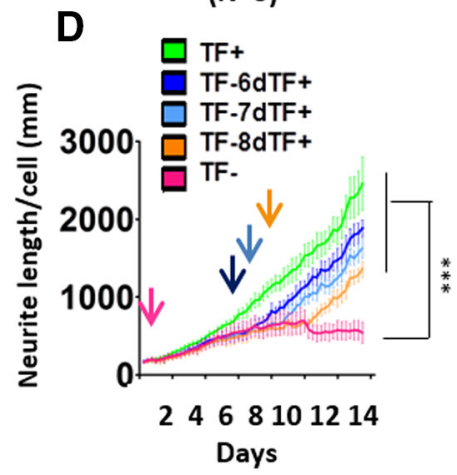

$(\mathrm{N}=5)$

E

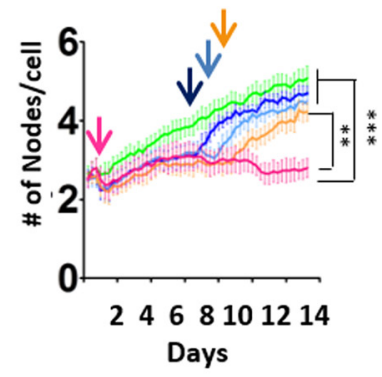

Days

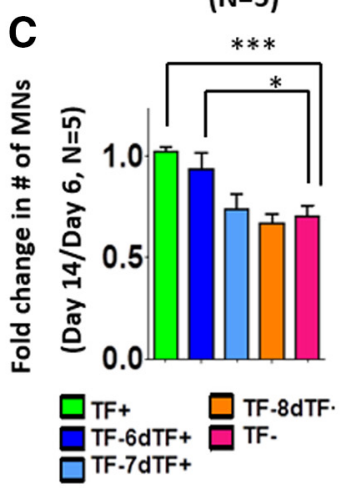

G

$\underline{\text { Kenpaullone }}$

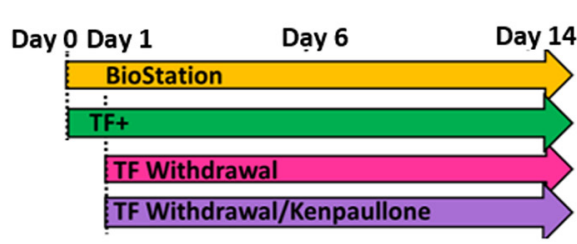

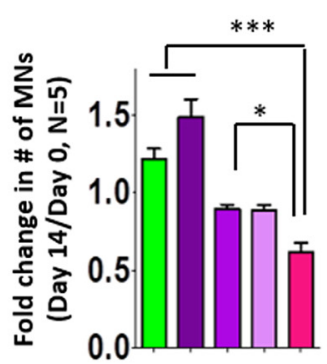

$\square$ TF+ $\mathrm{TF}+/ \mathrm{Ken} 5 \mathrm{uM}$
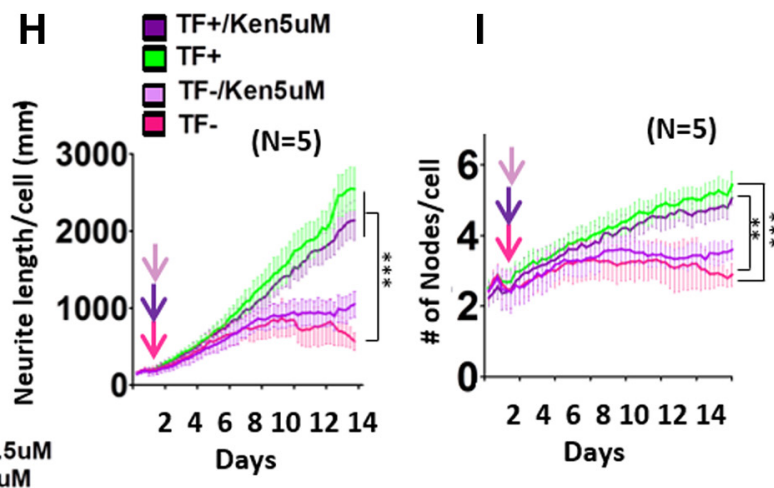

Figure 2. Measuring MN rescue responses following TF addback or kenpaullone treatment. $\boldsymbol{A}$, Experimental design of TF withdrawal and addback imaging assays. For all experiments, TF withdrawal began 1 day after the start of imaging defined as day 0 . For TF addback, the TFs- BDNF, GDNF, and $\mathrm{CNTF}^{-}$were reinstated to the culture media after 6,7 , or 8 days of TF withdrawal. $\boldsymbol{B}$, Representative images of Islet1::GFP MNs at day 1, 6, and 11 in $\mathrm{TF}^{+}$, TF addback at day 6 , and $\mathrm{TF}^{-}$. C, Fold change in number (\#) of MNs between day 6 and day 14 following TF addback at day 6,7 , or 8 . All data presented as mean $+\mathrm{SEM} . *, p<0.05 ; * * *, p$ $<0.001$ by $t$ test; all compared to $\mathrm{TF}^{-}$conditions $(n=5$ biological replicate experiments, each with three technical replicates). $\boldsymbol{D}, \boldsymbol{E}$, Time series plots depicting neurite length $(\boldsymbol{D})$ and number of nodes per cell $(\boldsymbol{E})$ show the MN population behavior in $\mathrm{TF}^{-}$and TF addback conditions. Key experimental time points are denoted with arrows: the start of TF withdrawal (red), day 6 addback (dark blue), day 7 addback (light blue), and day 8 addback (orange). Two-way repeated-measures ANOVA showed significant differences in neurite length per cell $(p<0.001, F=6.555, \mathrm{DF} n=4 ; \boldsymbol{D})$ and number of nodes $(p<0.05, F=3.356$, DFn $=4 ; \boldsymbol{E})$ over time under the different conditions. Subsequent Bonferroni post tests indicated TF addback at day 6, 7, and 8 increased neurite length per cell 
continued

$(\boldsymbol{D})$ and the number of nodes $(\boldsymbol{E})$ per cell compared to $\mathrm{TF}^{-}$at the end point. All data presented as mean $+\mathrm{SEM}$. **, $p<0.01 ; * * *$, $p<0.001$ by two-way repeated-measures ANOVA with Bonferroni correction, all compared to $\mathrm{TF}^{-}$conditions $(n=5$ biological replicate experiments, each with three technical replicates). $\boldsymbol{F}$, Experimental design of imaging assays kenpaullone addition to cells undergoing TF withdrawal. Analysis window between day 6 and day 14 was used for both evaluation of survival effect (G) and temporal quantitation of morphometric parameters $(\boldsymbol{H}, \boldsymbol{I})$. G. Fold change in \# of MNs between day 0 and day 14 of kenpaullone

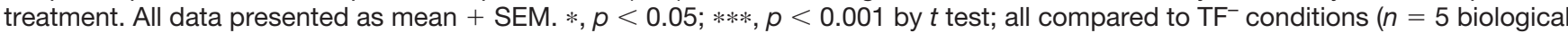
replicate experiments, each with three technical replicates). $\boldsymbol{H}, \boldsymbol{I}$, Time series plots depicting neurite length $(\boldsymbol{H})$ and number of nodes per cell $(I)$ after kenpaullone treatment. Kenpaullone (light and dark purple) was administered from the start of TF withdrawal (magenta) as denoted with arrows. All data presented as mean + SEM. **, $p<0.01 ; * * *, p<0.001$ by two-way repeated-measures ANOVA with Bonferroni correction, all compared to $\mathrm{TF}^{-}$conditions $(n=5$ biological replicate experiments, each with three technical replicates).

GFP expression is regulated by Islet1, an MN-specific transcription factor (Rigamonti et al., 2016). Preliminary experiments were performed to establish reproducible conditions for live-cell imaging. We tested several conditions including different MN differentiation protocols, seeding densities, and plating conditions. We selected a modified MN differentiation protocol (Yang et al., 2013; Qu et al., 2014), and plated MNs on astrocytes to prevent cells from clumping, which interfered with our ability to track single cells. To achieve high-resolution imaging without phototoxicity, we evaluated different illumination intensities and exposure times and used FluoroBrite DMEM medium to reduce background fluorescence. To initiate cell death in our cultures, we withdrew TFs (BDNF, GDNF, and CNTF) from culture medium. This is a wellestablished method to activate and study neuronal apoptosis (Yang et al., 2013). We used time-lapse live imaging to collect data at multiple time points over $\sim 2$ weeks. We then developed algorithms that enabled computational analysis of morphologic changes during the progressive stages of cell death (Videos 1-3).

\section{Identification of early-onset morphometric changes after TF withdrawal}

Neurons have a complex morphology, with many neurites (axons and dendrites) extending from the cell body (Fig. 1A). We used computational algorithms to analyze the number of MNs (Fig. 1B), the most commonly used survival parameter, and to quantify several key morphologic features, such as neurite length, number of nodes per cell, and cell body size (Fig. $1 C-E$ ). In the presence of TFs $\left(\mathrm{TF}^{+}\right)$, the number of $\mathrm{MNs}$ was relatively unchanged over time (Fig. 1B), while neurite length per cell (Fig. 1C), number of nodes per cell (Fig. 1D), and cell body size per cell (Fig. 1E) continued to increase. In contrast, after the removal of TFs $\left(\mathrm{TF}^{-}\right)$, neurite length per cell, number of nodes per cell, and cell body size per cell did not increase, and the number of MNs gradually decreased (Fig. 1B-E). Two-way repeated-measures ANOVA showed significant differences between $\mathrm{TF}^{+}$and $\mathrm{TF}^{-}$conditions over time in number of MNs, neurite length per cell, number of nodes per cell, and cell body size per cell over time (Fig. $1 B-E^{\mathrm{a}}$, Table $3^{\mathrm{a}}$ ). Subsequent Bonferroni posttests indicated that all the descriptors have significant differences between $\mathrm{TF}^{+}$and $\mathrm{TF}^{-}$conditions at the endpoint (Fig. 1B-E). Differences in the effect magnitudes in the number of nodes was striking compared to that in the number of $\mathrm{MNs}$ at day 12 (Fig. $1 F^{b}$ ).
We measured the fold change in the different morphometric parameters between days 1 and 12. Our results demonstrated that number of nodes per cell exhibited significant differential responses between $\mathrm{TF}^{+}$and $\mathrm{TF}^{-}$ conditions (Fig. 1G $; n=5, p<0.0001$ ). The greatest absolute difference was observed when measuring neurite length per cell; however, the node number measurement proved to be a more reproducible value between biological replicates and therefore had the lowest $p$-value. For this reason, we concluded that quantifying the number of nodes is a more sensitive indicator of the presence of TF support than measuring MN survival itself.

\section{Population-based analysis of the protective effects of TFs and kenpaullone}

We sought to define critical points in the neuronal degeneration and regeneration processes using population analysis of all cultured cells. We tracked and averaged the number of MNs, as well as their morphometric features, over time following TF withdrawal. We specifically aimed to determine for how long after TF withdrawal were MNs capable of being rescued by restoring TFs to the medium (TF addback), another paradigm that has been used for many years (Edwards et al., 1991). MNs underwent TF withdrawal for 6,7 , or 8 days, after which cells were either replenished with TF or maintained without trophic support (Fig. 2A, B). Tracking MN survival over time demonstrated that TF addback at day 6 was still capable of rescuing MNs (Fig. $2 C^{\text {d }} ; n=5, p<0.05$ ). At each time after TF withdrawal up to 8 days, TF addback increased neurite length per cell (Fig. $2 D^{\mathrm{e}} ; n=5, p<0.001$ ) and number of nodes per cell (Fig. $2 E^{\mathrm{e}} ; n=5$, TF addback at day 6 , day 7: $p<0.001$; TF addback at day 8: $p<0.01$ ), although the extent of recovery decreased with longer periods of TF withdrawal. Importantly, changes in these morphologic properties of MNs were again much more sensitive measures of cellular responses to the presence of TFs than was the number of cell bodies.

We also determined the effects of kenpaullone, an agent previously identified in an MN survival screen (Yang et al., 2013), using MNs deprived of TF. In this case, we treated MNs with two different concentrations of kenpaullone during the entire period in which they were maintained in the absence of TF (Fig. 2F). Under these conditions, we found that kenpaullone could preserve many of the MN cell bodies (Fig. 2G; $n=5, p<0.05$ ), and, to some degree, neurite length and the number of nodes, although this was not statistically significant (Fig. 

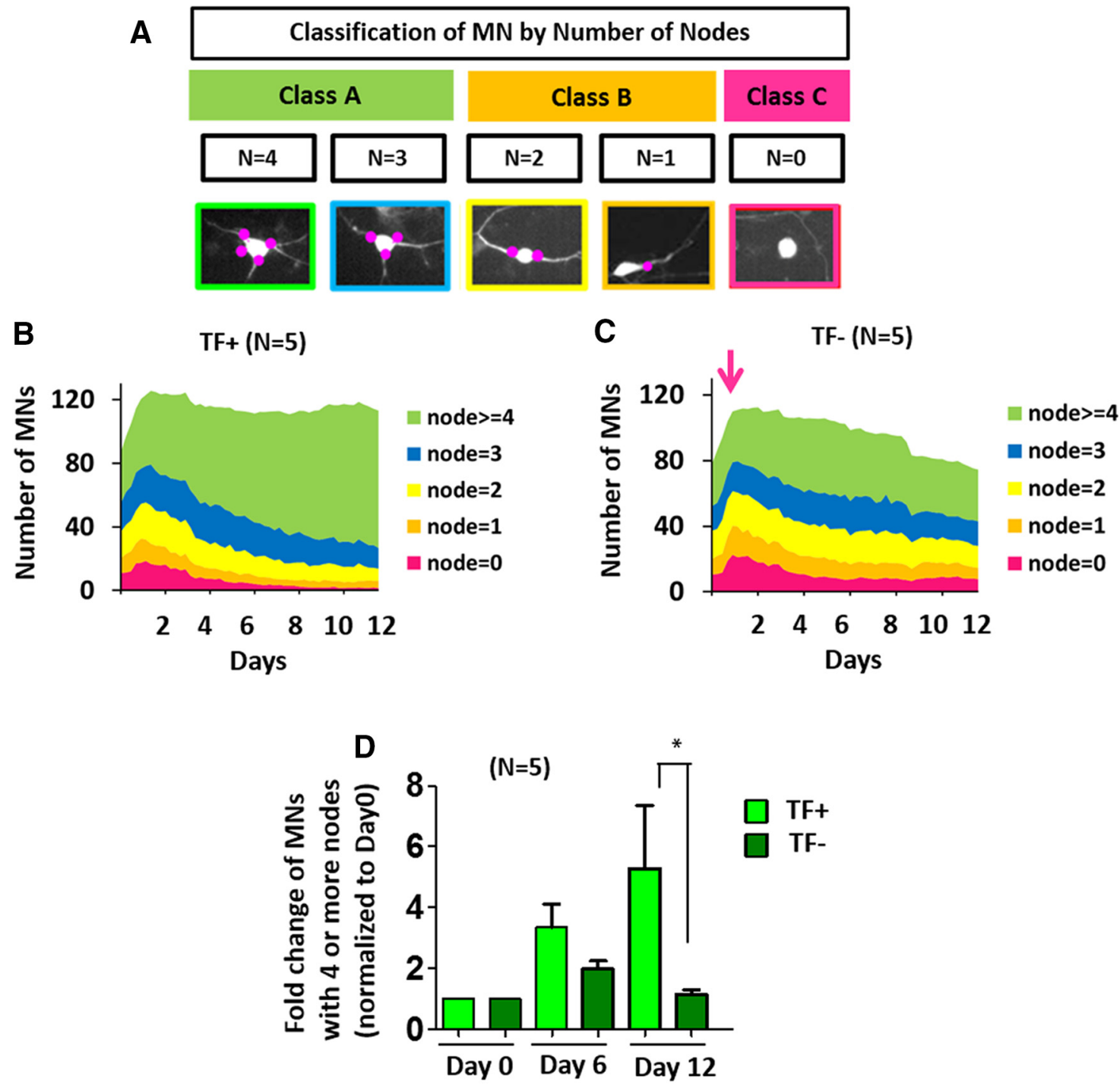

Figure 3. Classifying MNs according to their number of nodes. $\boldsymbol{A}$, Classification of the heterogeneous MN population according to their number of nodes $(0,1,2,3$ or $4+$ nodes) determines the class assignment (class $A, B, C)$ for each MN. B, C, A stacked temporal area plot displaying the number of MNs with $0,1,2,3$ or $4+$ nodes, quantified at each time point throughout the imaging experiment. In this stacked area graph, the height of each colored region on the $y$-axis indicates the number of MNs in each number of nodes category. (B), After the first few days in $\mathrm{TF}^{+}$, the total number of $\mathrm{MNs}$ remains relatively constant. MNs mature over time and eventually MNs with $4+$ nodes become the majority of the population. (C), In $\mathrm{TF}^{-}$, the total number of MNs gradually decreases over time and the MN population comprises a large percentage of cells with 2 or 3 nodes and relatively fewer neurons with 4 or more nodes. The magenta arrow indicates the time of TF withdrawal. $\boldsymbol{D}$, A histogram of fold change (relative to day 0 ) in the number of MNs with 4 or more nodes in the $\mathrm{TF}^{+}$and $\mathrm{TF}^{-}$conditions respectively. During the time period, the number of MNs with 4 or more nodes in $\mathrm{TF}^{+}$ and $\mathrm{TF}^{-}$conditions becomes increasingly different $(p<0.05, F=3.949$, DFn $=2$ by two-way repeated-measures ANOVA with Bonferroni correction). All data presented as mean + SEM. $*, p<0.05$. ( $n=5$ biological replicate experiments, each with three technical replicates.)

$\left.2 H, I^{9} ; n=5\right)$. Video 3 illustrates dynamic single-cell analysis of morphologic changes of MNs in different experimental conditions: TF, TF addback at day 6,5 $\mu \mathrm{M}$ kenpaullone, and TF withdrawal.

\section{Single-cell tracking-based parameters to evaluate the protective effects of TFs and kenpaullone}

Having quantified the responses of MN populations to varying TF conditions, we wanted to obtain additional information through tracking and quantifying the behaviors of individual cells such as number of nodes, cell body size, compactness, intensity, and velocity of their movement. One reason for doing this is that it has been demonstrated that distinct populations of cultured human MNs are much more prone to death then others (Arrasate and Finkbeiner, 2005; Skylaki et al., 2016; RodriguezMuela et al., 2017). First, we developed single-cell tracking metrics defining MN subpopulations according to their number of nodes (Videos 1 and 2). The number-of-nodes metric has several advantages that make it ideal for single-cell tracking. First, it correlates well with overall neuron health, since an increase or decrease in the num- 
A

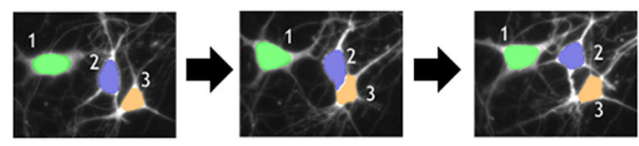

B

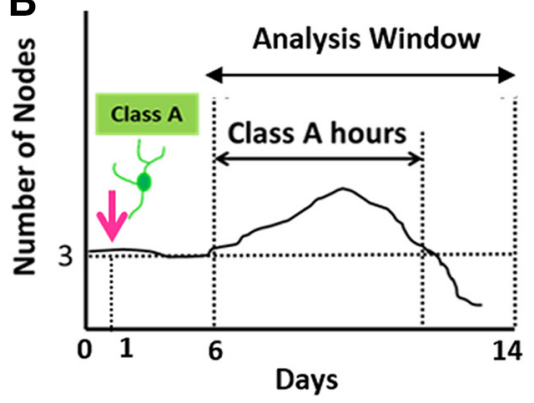

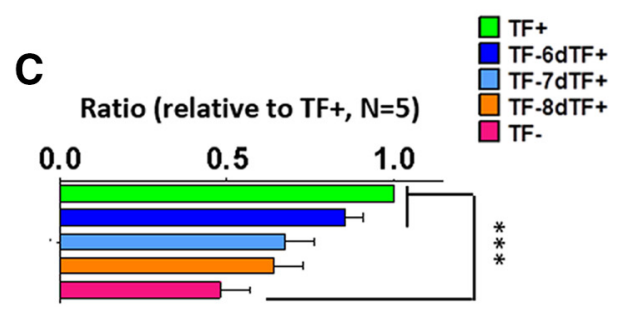

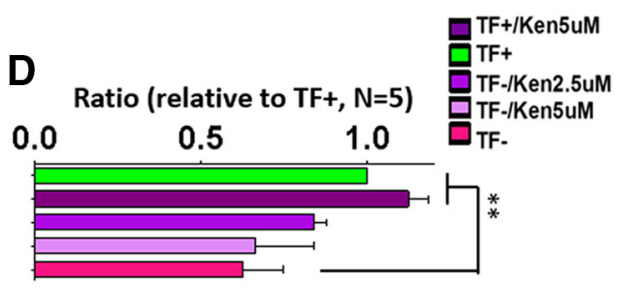

Figure 4. A Single-cell tracking algorithm to measure the lifespan of MNs. A, Representative images of cell tracking. Three MNs distinguished with different colors (green, purple, orange) were tracked by time lapse imaging using CL-Quant software The software was able to assign individual identities to two cells that were overlapping (middle image) using cell body size and position information collected over time. $\boldsymbol{B}$, Schematic diagram of the class A hours measurement. Class A MNs were defined at the beginning of the imaging period and then tracked. The same analysis window (between day 6 and day 14) was used for all experiments. Using single-cell tracking with a mask for node number, the lifespan hours of class A MNs were measured and averaged during the selected analysis window. For each MN, class A hours ended when class A MNs changed to class B or C. C, D, Normalized class A hours (relative to $\mathrm{TF}^{+}$) for TF addback $(\boldsymbol{C})$ and kenpaullone $(\boldsymbol{D})$. Table 1 lists the number of tracked MNs for the TF addback experiments, and Table 2 provides the numbers of tracked MNs for the kenpaullone experiments. Data presented as mean $+\mathrm{SEM}$. $* *, p<0.01$; $* * *, p<0.001$ by $t$ test all compared to $\mathrm{TF}^{-}(n=5$ biological replicate experiments, each with three technical replicates).

ber of nodes in MN corresponded to both TF withdrawal and rescue responses. Second, the number of nodes could be readily tracked by live-cell imaging at the singlecell level (Video 2). Representative tracking analysis of neurite length (yellow), number of nodes (magenta), and cell body size (green) of MNs in four experimental conditions is shown in Video 3. In $\mathrm{TF}^{+}$(Video $3 A$ ), individual MNs showed increased neurite length and number of nodes. TF addback at day 6 (Video $3 B$ ) rescued both neurite length and number of nodes. $5 \mu \mathrm{M}$ kenpaullone (Video $3 C$ ) was less protective compared to TF addback (Video 3B). After TF withdrawal (Video 3D), the neurites retracted and the cell body size and number of nodes decreased.

Thus, we divided MNs into five categories according to their number of nodes ( $n=0,1,2,3$, >4; Fig. $3 A$ ) and quantified the number of $\mathrm{MNs}$ in each category over time (Fig. 3B, C). Comparing MN compositions between $\mathrm{TF}^{+}$ (Fig. $3 B$ ) and $\mathrm{TF}^{-}$(Fig. $3 C$ ) conditions revealed striking differences. In $\mathrm{TF}^{+}$, MNs with 4 or more nodes were the most abundant population and increased throughout the imaging period, while the number of $\mathrm{MNs}$ in the other categories decreased (Fig. 3B). This reflects the natural maturation of MNs developing a more complex neurite network. However, in $\mathrm{TF}^{-}$, the population of MNs with 4 or more nodes was significantly smaller and did not continue to increase over time (Fig. $3 C$ ). In the $\mathrm{TF}^{-}$condition, the proportion of each type of $\mathrm{MN}$ remained fairly constant throughout, with a slight decrease of $\mathrm{MNs}$ in all categories over time, indicating cell death that accompanies TF with- drawal (Fig. 3C). The number of MNs with 4 or more nodes became statistically significant between $\mathrm{TF}^{+}$and $\mathrm{TF}^{-}$conditions over time (Fig. $3 D^{\mathrm{h}} ; n=5, p<0.05, F=3.949$, DFn = 2). At the end of the analysis (day 12), the number of MNs with 4 or more nodes was significantly higher in $\mathrm{TF}^{+}$compared to $\mathrm{TF}^{-}$(Fig. $3 D^{\mathrm{h}} ; n=5, p<0.05$ ).

We next collected data on single MNs classified according to their number of nodes: class $A$ (three or more nodes; the most well-developed cells), class B (one or two nodes), and class $C$ (no nodes). We wanted to determine if neurons with more nodes were more likely to survive TF withdrawal than neurons with fewer nodes. We started by individually tracking the MNs with the most nodes (class A). We defined a metric, "class A hours," as the length of time in which MNs remained as class A (Fig. 4B). After TF withdrawal, class $A$ MNs transitioned into class $B$ or $C$, resulting in a shorter class $A$ hours period. However, TF addback at day 6 significantly increased class $A$ hours (Fig. $4 C^{i} ; n=5, p<0.001$ ). TF addback at days 7 and 8 appeared to increased class A hours somewhat as well, but those effects were not statistically significant. Next, we used the same class $A$ hour measurement to evaluate kenpaullone's effects on MNs, using the same experimental conditions as in Fig. 4D. Kenpaullone-treated MNs showed a non-statistically significant trend toward increasing class $A$ hours of MNs undergoing TF withdrawal (Fig. $4 D^{\mathrm{j}} ; n=5$ ).

We next sought to examine the morphometric changes of individual MNs over time after TF withdrawal using computational single-cell tracking. We could not track 

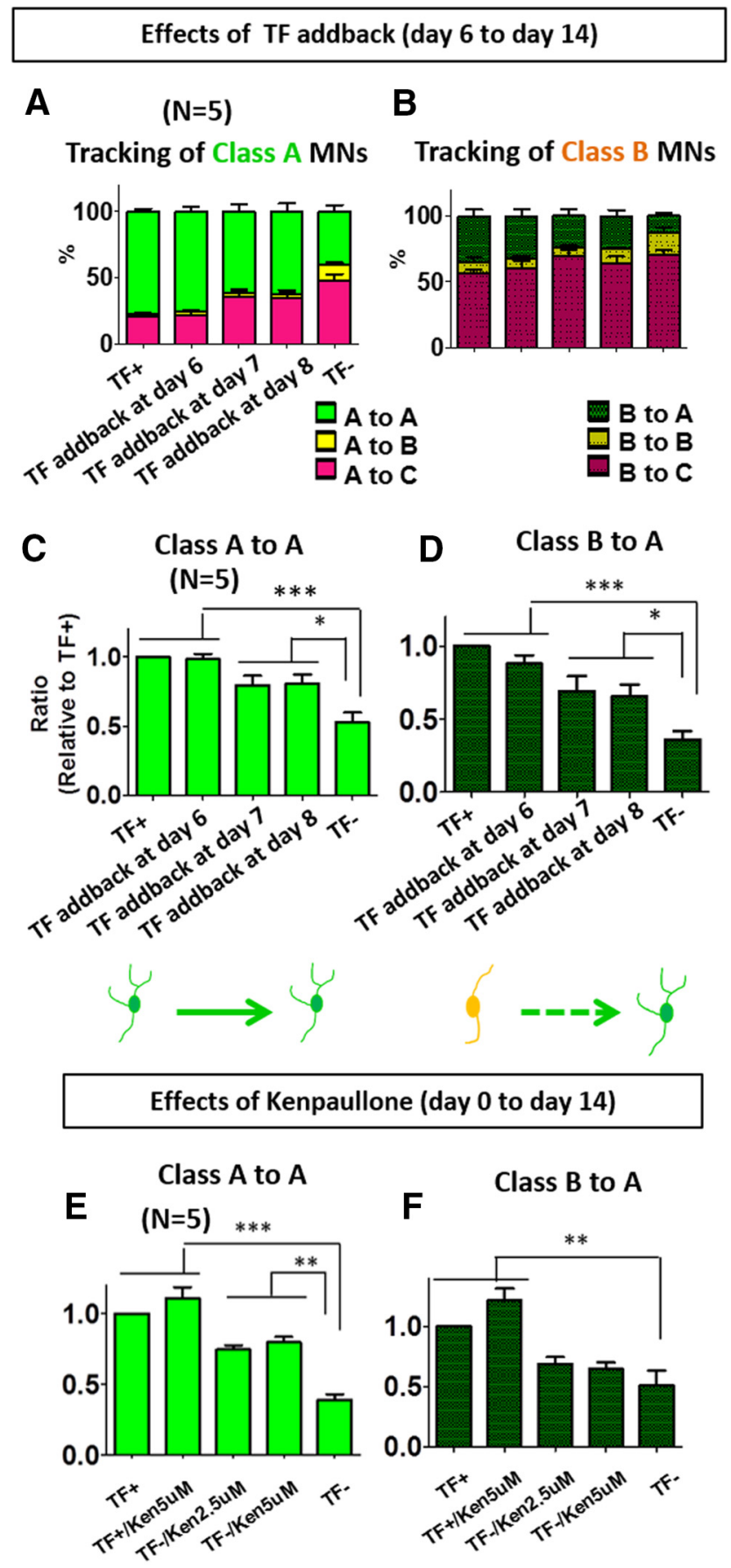

Figure 5. Tracking cell class transitions of individual MNs in TF withdrawal, TF addback, and kenpaullone conditions. Cells were categorized as either class $A$ or class $B M N$ as shown in Fig. $3 A$ and then individually tracked to determine if they remained in the same class at the end of the analysis window. Table 1 details the class transitions for all tracked MNs in the TF addback experiments, while Table 2 provides this information for the kenpaullone experiments. $\boldsymbol{A}, \mathrm{MNs}$ identified as class A on day 6 were tracked until day 14 in each treatment condition and were categorized according to their class transition in a stacked histogram ( $n=5$ biological replicate experiments, each with three technical replicates). $\boldsymbol{B}$, Stacked histogram of class transitions from day 6 to day 14 of class B MNs in each treatment condition ( $n=5$ biological replicate experiments, each with three technical

\section{Figure 5. continued}

replicates). $\boldsymbol{C}$, The ratio of class $A$ to $A$ MNs (from day 6 to day 14 ) in each different treatment condition, relative to $\mathrm{TF}^{+}$control, quantifies the maintenance of class A MNs. TF addback at day 6 shows a significant rescue effect, while TF addback at day 7 or 8 is less effective. Data presented as mean + SEM. $*, p<0.05$; ***, $p<0.001$ by $t$ test; all compared to $\operatorname{TF}^{-}(n=5$ biological replicate experiments, each with three technical replicates). $\boldsymbol{D}$, The ratio of class $B$ to $A$ MNs (from day 6 to day 14) in the different treatment conditions relative to $\mathrm{TF}^{+}$quantifies the rescue of class B MNs to class A MNs. Data presented as mean + SEM. $*, p<0.05 ; * * *, p<0.001$ by $t$ test; all compared to $\mathrm{TF}^{-}$ ( $n=5$ biological replicate experiments, each with three technical replicates). $\boldsymbol{E}$, Maintenance of class A to A MNs from day 0 to day 14 in different treatment conditions relative to $\mathrm{TF}^{+}$. Kenpaullone significantly maintained class A MNs as class A compared to TF withdrawal. Data presented as mean + SEM. $* *, p<0.01$; $* * *, p<0.001$ by $t$ test; all compared with $\mathrm{TF}^{-}$conditions $(n=$ 5 biological replicate experiments, each with three technical replicates). $\boldsymbol{F}$, Changes in the ratio of class $B$ to $A$ MNs from day 0 to day 14 . Kenpaullone did not rescue many class $B$ MNs to class A. Data presented as mean + SEM. **, $p<0.01 ; * * *, p<$ 0.001 by $t$ test; all compared with $\mathrm{TF}^{-}$conditions $(n=5$ biological replicate experiments, each with three technical replicates).

class C MNs, as they quickly died and disappeared from view. Thus, we tracked the individual fate transitions within and between class A (Fig. $5 A$ ) and class B (Fig. 5B) MNs by measuring changes in the number of nodes per cell. We found that two cell fate transitions-class $A$ to $A$ (Fig. 5C, E) and class $B$ to $A$ (Fig. 5F)-were useful in evaluating $M N$ rescue effects. Most class $A$ MNs remained in class $A$ (class $A$ to $A$ ) after TF addback at day 6 , 7, and 8 (Fig. $5 C^{\mathrm{k}} ; n=5$, TF addback at day $6: p<0.001$, TF addback at day 7: $p<0.05$, TF addback at day $8: p<$ $0.05)$. Similarly, tracking class $B$ MNs indicated that more class $B$ MNs were rescued to class $A$ MNs (class $B$ to $A$ ) after TF addback at day 6,7 , and 8 compared to the TF withdrawal condition, with the largest rescue occurring at day 6 (Fig. $5 D^{\prime}$; TF addback at day $6: p<0.001$, TF addback at day 7: $p<0.05$, addback at day 8: $p<0.05$ ). We also tested the effects of kenpaullone under the same conditions as in Fig. 2F. Again, we found that this compound could maintain class A MNs (Fig. $5 E^{\mathrm{m}} ; n=5, p<$ 0.01 ), but in contrast to TF addback, kenpaullone did not cause class B MNs to develop into class A MNs (Fig. $5 F^{n}$; $n=5$ ). Meanwhile adding kenpaullone at day 6 did not improve survival (data not shown). Tables 1 and 2 show MN numbers for each category.

\section{Reverse tracking analysis elucidates key features of rescuable class $B$ MNs}

Results from the previous section established that many class A MNs could retain class A features after both TF addback and kenpaullone treatment, but only TF could permit class B MNs to mature into class A MNs. However, not all class $B$ MNs were rescued to class $A M N s$, even after TF addback. Fig. $6 A$ and $B$ shows representative single-cell analyses for number of nodes and cell body size. Both $\mathrm{MN} \# 1$ and $\mathrm{MN} \# 2$ "regressed" into class B after TF withdrawal at day 6 , but only $\mathrm{MN} \# 1$ recovered to class $A$ after TF addback (Fig. $6 A$ ). We next defined the 


\section{Single cell tracking analysis}
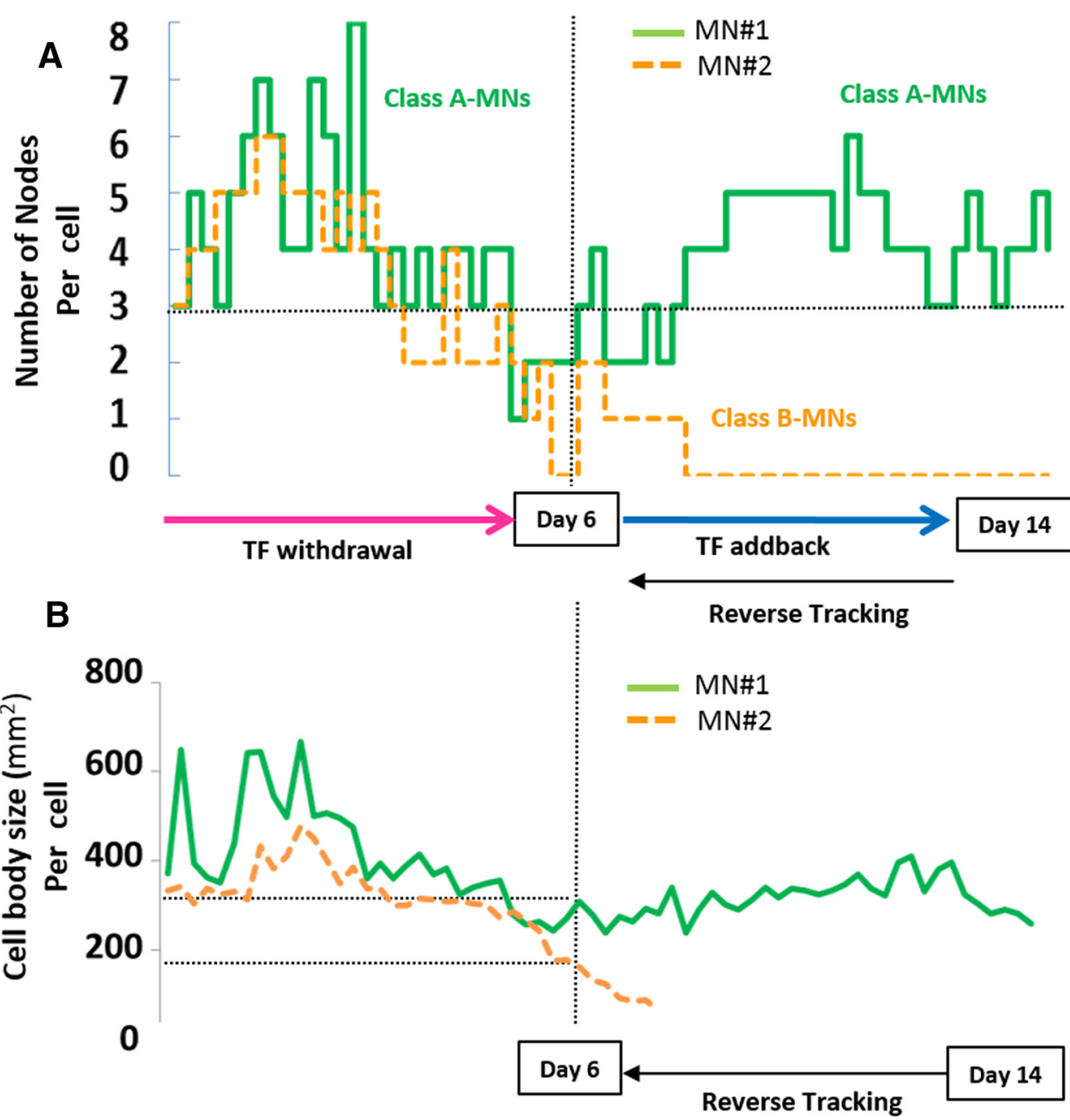

Common features of rescuable Class B- MNs at day 6, day 7, and day 8

C

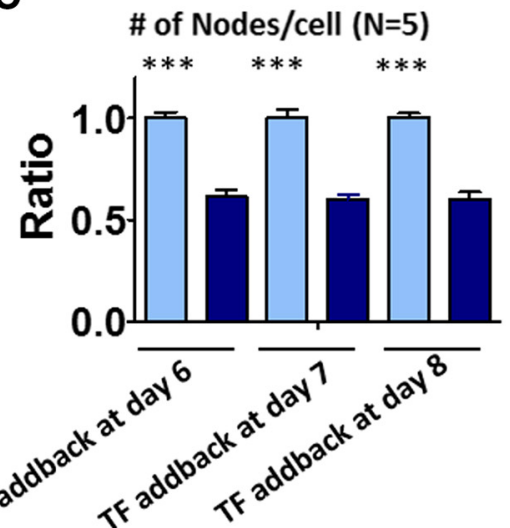

D Cell body size/cell

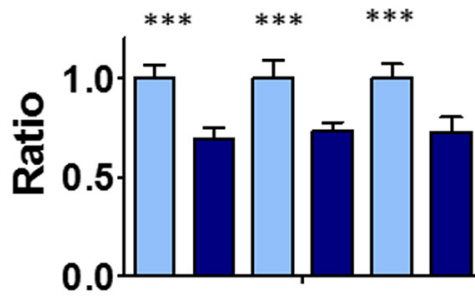

口 Rescuable Class B-MNs

Unrescuable Class B-MNs

Figure 6. Characterization of key morphologic features of rescuable class $B$ MNs using reverse tracking. $\boldsymbol{A}, \boldsymbol{B}$, Representative single cell analysis extracted using CL-Quant. Graphs show tracked number of nodes $(\boldsymbol{A})$ and cell body size $(\boldsymbol{B})$ of two individual MNs over time. In this plot, both MN \#1 and MN \#2 became class B MNs after TF withdrawal at day 6 . MN \#1 recovered to class A following day 6 of TF addback. $\boldsymbol{C}, \boldsymbol{D}$, Common features of rescuable class B MNs at day 6,7 and 8 . At the time point immediately before TF addback, class B MNs that could develop into class A MNs had more nodes $(\boldsymbol{C})$ and a larger cell body size $(\boldsymbol{D})$ compared to class 
continued

B MNs that could not regrow neurites. Data presented as mean + SEM. $* * *, p<0.001$ by $t$ test; all compared with TF $^{-}$conditions ( $n=5$ biological replicate experiments, each with three technical replicates).

characteristics of class $B$ MNs that predicted their ability to be fully rescued by TF addback. First, we identified individual class $B$ MNs that either were or were not rescued after TF addback at day 6,7 , or 8 . Then, we reversetracked the MNs back to the time of TF withdrawal and examined key morphologic parameters, such as number of nodes (Fig. 6C), cell body size (Fig. 6D), compactness, and velocity (data not shown) at day 6,7 , and 8 . This analysis established that the class B MNs that could recover to class A MNs after TF addback had more nodes (Fig. $6 C^{\circ} ; n=5, p<0.001$ ) and a larger cell body (Fig. $\left.6 D^{\mathrm{p}} ; n=5, p<0.001\right)$ compared to class $\mathrm{B}$ MNs that did not recover. Thus, MNs with more nodes and larger cell bodies are the subset of MNs that are most likely to recover after a death stimulus, whereas class C MNs cannot be rescued with any of the treatments or compounds we used.

\section{Discussion}

Live-cell imaging of individual cells may be much more informative than population-based methods of studying cell behavior (Arrasate and Finkbeiner, 2005; Sharma et al., 2012). This is particularly true in stem cell-derived neuronal cell cultures that typically are quite variable in composition. However, it can be somewhat onerous to carry out and is normally quite limited in terms of the numbers of cells that can be analyzed (Skibinski and Finkbeiner, 2013; Skylaki et al., 2016). In this paper, we used the BioStation CT that was developed to facilitate prolonged visualization of cells. Analysis of cells using this instrument in combination with advanced analysis software provided us with the ability to computationally identify, categorize, and track individual cells. Our work shows that this approach provides detailed measurements of morphologic changes that occur in individual MNs over time as they degenerate.

The software we employed is highly customizable, so any morphologic attribute can be selected to categorize and track cell outcomes. Importantly, these timedependent measurements do not necessarily need to be predefined. Because each cell is individually tracked, its "lifetime" can be computationally annotated by specific morphologic features. This ability is especially relevant to in vitro-derived cultures, because the terminally differentiated cells of interest arise and mature at different times. We used the morphometric analysis assay to detect distinct modes of action underlying MN rescue seen with TF addback versus kenpaullone treatment. While both treatments were efficacious, only TF addback restored class $B$ MNs to class A. In contrast, kenpaullone primarily acted to improve survival of the class A MN population. The robust, beneficial effect of TF addback is consistent with reports that TFs can salvage unhealthy neurons in animal models of ALS and have a neuroprotective effect on axotomized MNs (Gimenez y Ribotta et al., 1997; Sendtner et al., 1997).
Changes that occur early in neurodegenerative processes might provide key, clinically relevant insights into how to identify cells that possess the capacity for full functional restoration. In this study, we showed that MNs exposed to stress-inducing conditions rapidly exhibited changes in their neurite length and number of nodes. Changes in these parameters appeared to occur early in the death process and constituted sensitive indices of neuronal health. Neurite retraction and reduced soma size are known to accompany neurodegeneration, even in patients (Kiernan and Hudson, 1993; Anglade et al., 1997; Takeda et al., 2014). We therefore divided MNs into classes based on their number of nodes and used singlecell tracking to examine changes in node number and correlate them with survival outcomes. We observed that neurons with more nodes had a higher survival rate. We further found that cell body size is another strong predictor of survival outcome. However, of these two early morphologic events, the number of nodes is the more feasible to track temporally in single cells. Our findings illustrate the utility of novel analytical approaches that can be applied to live-cell imaging data sets, especially when compared to more conventional types of survival assays.

Our live-cell analysis platform possesses flexibility and analytical power, making it broadly applicable to a wealth of cell biology inquiries. Live-cell analysis of individual cells is also strongly complemented by the current growing trend in single-cell RNA sequencing (Chen and Arlotta, 2016; Shekhar et al., 2016; Zheng et al., 2017). These technologies can be used to study cellular processes that occur during disease progression and determine how treatments affect these processes or others that underlie disease states and precede cell death. These studies may also help elucidate ideal time points for optimal therapeutic intervention. Hopefully, observations like these will lead to the development of a new generation of therapeutics capable of truly reversing degenerative phenotypes.

\section{References}

Anglade P, Vyas S, Javoy-Agid F, Herrero MT, Michel PP, Marquez J, Mouatt-Prigent A, Ruberg M, Hirsch EC, Agid Y, (1997) Apoptosis and autophagy in nigral neurons of patients with Parkinson's disease. Histol Histopathol 12:25-31. Medline

Arrasate M, Finkbeiner S, (2005) Automated microscope system for determining factors that predict neuronal fate. Proc Natl Acad Sci U S A 102:3840-3845. CrossRef Medline

Brennand KJ, et al. (2015) Creating patient-specific neural cells for the in vitro study of brain disorders. Stem Cell Reports 5:933-945. CrossRef Medline

Chen HH, Arlotta P, (2016) Seq-ing the cortex one neuron at a time. Nat Neurosci 19:179-181. CrossRef Medline

Davies CA, Mann DM, Sumpter PQ, Yates PO, (1987) A quantitative morphometric analysis of the neuronal and synaptic content of the frontal and temporal cortex in patients with Alzheimer's disease. $J$ Neurol Sci 78:151-164. CrossRef

Edwards SN, Buckmaster AE, Tolkovsky AM, (1991) The death programme in cultured sympathetic neurones can be suppressed at 
the posttranslational level by nerve growth factor, cyclic AMP, and depolarization. J Neurochem 57:2140-2143. Medline

Gimenez y Ribotta M, Revah F, Pradier L, Loquet I, Mallet J, Privat A, (1997) Prevention of motoneuron death by adenovirus-mediated neurotrophic factors. J Neurosci Res 48:281-285. Medline

Grskovic M, Javaherian A, Strulovici B, Daley GQ, (2011) Induced pluripotent stem cells-opportunities for disease modelling and drug discovery. Nat Rev Drug Discov 10:915-929. CrossRef Medline

Kiernan JA, Hudson AJ, (1993) Changes in shapes of surviving motor neurons in amyotrophic lateral sclerosis. Brain 116: 203-215. CrossRef

Qu Q, Li D, Louis KR, Li X, Yang H, Sun Q, Crandall SR, Tsang S, Zhou J, Cox CL, Cheng J, Wang F, (2014) High-efficiency motor neuron differentiation from human pluripotent stem cells and the function of Islet-1. Nat Commun 5:3449. CrossRef Medline

Rigamonti A, Repetti GG, Sun C, Price FD, Reny DC, Rapino F, Weisinger K, Benkler C, Peterson QP, Davidow LS, Hansson EM, Rubin LL, (2016) Large-scale production of mature neurons from human pluripotent stem cells in a three-dimensional suspension culture system. Stem Cell Reports 6:993-1008. CrossRef

Rodriguez-Muela N, Litterman NK, Norabuena EM, Mull JL, Galazo MJ, Sun C, Ng SY, Makhortova NR, White A, Lynes MM, Chung WK, Davidow LS, Macklis JD, Rubin LL, (2017) Single-cell analysis of SMN reveals its broader role in neuromuscular disease. Cell Rep 18:1484-1498. CrossRef Medline

Sendtner M, Gotz R, Holtmann B, Thoenen H, (1997) Endogenous ciliary neurotrophic factor is a lesion factor for axotomized motoneurons in adult mice. J Neurosci 17:6999-7006. Medline
Sharma P, Ando DM, Daub A, Kaye JA, Finkbeiner S, (2012) Highthroughput screening in primary neurons. Methods Enzymol 506: 331-360. CrossRef Medline

Shekhar K, Lapan SW, Whitney IE, Tran NM, Macosko EZ, Kowalczyk M, Adiconis X, Levin JZ, Nemesh J, Goldman M, McCarroll SA, Cepko CL, Regev A, Sanes JR, (2016) Comprehensive classification of retinal bipolar neurons by single-cell transcriptomics. Cell 166:1308-1323.e1330. CrossRef

Skibinski G, Finkbeiner S, (2013) Longitudinal measures of proteostasis in live neurons: features that determine fate in models of neurodegenerative disease. FEBS Lett 587:1139-1146. CrossRef Medline

Skylaki S, Hilsenbeck O, Schroeder T, (2016) Challenges in longterm imaging and quantification of single-cell dynamics. Nat Biotechnol 34:1137-1144. CrossRef Medline

Takeda T, Uchihara T, Nakayama Y, Nakamura A, Sasaki S, Kakei S, Uchiyama S, Duyckaerts C, Yoshida M, (2014) Dendritic retraction, but not atrophy, is consistent in amyotrophic lateral sclerosiscomparison between Onuf's neurons and other sacral motor neurons. Acta Neuropathol Commun 2:11. CrossRef Medline

Yang YM, Gupta SK, Kim KJ, Powers BE, Cerqueira A, Wainger BJ, Ngo HD, Rosowski KA, Schein PA, Ackeifi CA, Arvanites AC, Davidow LS, Woolf CJ, Rubin LL, (2013) A small molecule screen in stem-cell-derived motor neurons identifies a kinase inhibitor as a candidate therapeutic for ALS. Cell Stem Cell 12:713-726. CrossRef Medline

Zheng GX, et al. (2017) Massively parallel digital transcriptional profiling of single cells. Nat Commun 8:14049. CrossRef Medline 